\title{
Role of Some Risk Factors in the Etiology of Breast Cancer in the Sudan
}

\author{
Hussain Gadelkarim Ahmed ${ }^{*}, 1$, Rehab Mohammed Musa ${ }^{2}$, Mohmmed Madani Eltybe ${ }^{3}$ and \\ Mohmmed Omer Mohmmed Hussein ${ }^{4}$
}

\author{
${ }^{I}$ Department of histopathology and Cytopathology, FMLS, University of Khartoum, Khartoum, Sudan \\ ${ }^{2}$ Department of Clinical pharmacology, FP, University of Khartoum, Khartoum, Sudan \\ ${ }^{3}$ Faculty of medical laboratory Science, Elneelain University, Khartoum, Sudan \\ ${ }^{4}$ Almoulem Medical centre, Khartoum, Sudan
}

\begin{abstract}
Aim: The aim of this study was to correlate breast cancer by common breast cancer risk factors in the Sudan.
Methodology: Using a purposeful questionnaire 150 female breast cancer patients and 100 apparently health controls were asked detailed information on about risk factors.

Results: Out of the 150 women with breast cancer, 38 (25.3\%), 22(14.7\%), 26(17.3\%), 20(13.3\%), 21(14\%), 11(7.3\%), $72(48 \%)$, and $86(57.3 \%)$ were identified as having a previous history of oral contraceptives usage, a family history of breast cancer, a past history of benign breast disease, a previous history of breast cancer, a previous breast biopsy, claimed other cancers in their families, confessed a pesticides exposure, and over weigh in most of their lives respectively. Out of the 150 women, $44(29.3 \%$ ) were found to have a previous physical activity. Moreover, $44.8 \%$ and $66.9 \%$ have attended the menarge at the age of 13 and 14 respectively. Of these factors, statistical significant risks were found with, past history of benign breast disease $(\mathrm{P}<0.04)$, previous breast biopsies $(\mathrm{P}<0.07)$, pesticides and plasticizers exposure $(\mathrm{P}<0.01$ and $0.04)$, period of being over weight $(\mathrm{P}<0.001)$, practice physical activities $(\mathrm{P}<0.0001)$, unmarried $(\mathrm{P}<0.002)$, decreased number of children $(\mathrm{P}<0.002)$. According to the ethnic group, Gaalyaeen tribes represented $61(40.7 \%)$ of the study subjects. Most of the patients were from Khartoum state, constituting 37.3\%.

Conclusion: There was variable exposure to many risk factors for breast cancer in the Sudan. The study suggests further separate measurement of risk factors for breast cancer, as well as, factors that might reduce those risk factors. Cohort mean of evaluation is highly recommended.
\end{abstract}

Keywords: Breast cancer, risk factors, Sudan, etiology.

\section{INTRODUCTION}

Female breast cancer is by far the leading cancer in the Sudan. The alarmingly high frequency of women presenting with advanced breast cancer to the Radiation Isotope Center Khartoum (RICK) and Gaziera Institute for Cancer treatment and Molecular Biology (GICMB), which are the only two oncology centers in the Sudan, has prompted looking for an investigation that might help in solving this real health problem. The highest percentages were recorded in 1998 (38.4\% of all female cancers), followed by the years, 2000 , 1999 and 2001, which attended $36.03 \%, 35.2 \%$ and $32.4 \%$ respectively. Furthermore, the records from GICMB indicated the highest percentages (42\%) of breast cancer [1].

Risk factors for breast cancer are important for a reduction of the incidence of the disease if avoided and enable better prediction of risk and targeting of preventive measures if are not avoidable.

Breast cancer, like other forms of cancer, is considered to result from multiple environmental and hereditary factors, as

*Address correspondence to this author at the University of Khartoum, 102, Toombak and Smoking Research Centre, Khartoum, Sudan; Tel: +249155130423; E-mail: Hussaingad1972@yahoo.com well as, lesions result from DNA changes such as genetic mutations. Exposure to estrogen has been experimentally linked to the mutations that causes breast cancer [2]. Beyond the contribution of estrogen, research has implicated viral oncogenesis and the contribution of ionizing radiation. However, many factors have been indicated to contribute to the etiology of breast cancer. a) Failure of immune surveillance, which usually removes malignancies at early phases of their natural history. b) Abnormal growth factor signaling in the interaction between stromal cells and epithelial cells, for example in the angiogenesis necessary to promote new blood vessel growth near new cancers. c) Inherited defects in DNA repair genes, such as BRCA1, BRCA2 and p53.

Although many epidemiological risk factors have been identified, the cause of any individual breast cancer is often unknowable. In other words, epidemiological research informs the patterns of breast cancer incidence across certain populations, but not in a given individual. Approximately $5 \%$ of new breast cancers are attributable to hereditary syndromes, while no etiology is known for the other $95 \%$ of cases [3].

Breast cancer represents a major health problem in the Sudan and the number of new cases escalating each year. 
However, Sudan is a very large country, inhibited by many different heterogeneous communities. These communities greatly varying in their life style and exposure to environmental factors that might be potent risk factors for the development of breast cancer. What is more, no epidemiological study has undertaken this issue before. There-fore, this study is an attempt to identify the most frequent risk factors, which might contribute to the etiology of breast cancer in the Sudan.

\section{METHODOLOGY}

In this case control study, risk factors for breast cancer were evaluated among 150 women with breast cancer (ascertained as cases) and 100 apparently health women (ascertained as controls); their ages ranging from 20 to 65 years with a mean age of 40 years old. The majority of patients were at the age range $36-45$ years constituting $60(40 \%)$; hence the distribution was similar in respect to the upper and lower limits from the mean. Using a purposeful questionnaire 250 study subjects were asked detailed information on family history of cancer and reproductive history (age at menarge, marital status, age at first live birth, number of pregnancies, number of children, age at menopause, family history of breast cancer, past history of benign breast disease, previous history of breast cancer and previous breast biopsy. Additional information was collected on hormone use (oral contraceptives (OC), pesticides and plasticizers exposure and demographics (age, at breast cancer diagnosis, residence and race/ethnicity). Physical activity and self-reported height and weight for at least one year before diagnosis were obtained to calculate body mass index (BMI, $\mathrm{kg} / \mathrm{m} 2$ ).

Sample Size: Sample size (150) was calculated using software Known as the survey system available at http:/www.surveystem.com.sscalc.htm. The system inertly relies on this equation: $\mathrm{n}=\mathrm{z} 2 \mathrm{pq} / \mathrm{d} 2(\mathrm{n}=$ sample size; $\mathrm{z}=$ the standard normal deviate, usually set at 1.96 , which corresponds to the level of the $95 \%$ confidence level; $p=$ the proportion to the target population i.e., percentage of the studied group, it is 0.11 in this study; $\mathrm{q}=1.0-\mathrm{p}$.

Statistical Analysis: Data management was done using Statistical Package for Social Sciences (SPSS version 12). SPSS was used for analysis and to perform Pearson Chisquare test for statistical significance ( $\mathrm{P}$ value). The 95\% confidence level and confidence intervals were used.

Ethical Consent: Each participant was asked to sign a written ethical consent during the interview in addition to informed ethical form was designed and passed the ethical committee of the faculty research board.

\section{RESULTS}

Out of the 150 patients with breast cancer (cases), and 100 apparently healthy individuals (controls); 38 (25.3\%) and $38(38 \%)$ were identified as having a previous history of oral contraceptives usage, respectively. Information concerning the type of oral contraceptives were available for only 35 patients, of whom $28(80 \%)$ were using progesterone only pill and the remaining seven $(20 \%)$ were using combined pill.
Out of the 150 cases and 100 controls, $22(14.7 \%)$ and $14(14 \%)$ were found with a family history of breast cancer (First degree mother side), as well as, 20 (13.3\%) were detected as having a previous history of breast cancer, respectively. Furthermore, 11(7.3\%) of the cases and 27 $(27 \%)$ of the controls have claimed other cancers in their families.

Moreover, $26(17.3 \%)$ and $8(8 \%)$ of cases and controls have revealed a past history of benign breast disease $(\mathrm{P}<$ $0.04)$, in addition to $21(14 \%)$ and $7(7 \%)$ of the cases and controls have revealed that they have done previous breast biopsies $(\mathrm{P}<0.07)$.

In regard to the exposure to pesticides and plasticizers, $72(48 \%)$ and $65(43.3 \%)$ of the cases and $30(30 \%)$ and $54(54 \%)$ of controls have confessed exposure to pesticides and plasticizers, respectively. The risks associated with pesticides and plasticizers were significant $(\mathrm{P}<0.01$ and $0.04)$, respectively.

According to the body weight, of the total 150 women, $86(57.3 \%)$ have confessed their being over weight in most of their lives, hence, the remaining $64(42.7 \%)$ have claimed a normal body weight through out their lives. Out of 86 $(100 \%)$ over weighted women, $48(32 \%), 3(2 \%)$ and 35 (23.3\%) were being over weight for periods of 20+, 10-19 and less than 10 years respectively, as indicated in Fig. (1). Of the 100 controls, $64(64 \%)$ claimed over weight. However, the relation between cases and controls regarding over eight, in general was not found to be statistically significant.

Nevertheless, most of those over weighted for a period of less than 10 years were relatively younger. Of the total 35 $(100 \%)$ being over weighted for less than 10 years, 32 (91.4\%) were younger than the age of 45 years, hence, the majority of those being over weighted for more than 20 years were older. Of the total $48(100 \%)$ being over weighted for more than 20 years, $33(68.8 \%)$ were older than the age of 45 yeas. The relationship between the period of being over weighted and risk of breast cancer was found to be statistically significant $(\mathrm{P}<0.001)$.

Practicing irregular physical activities was found among $44(29.3 \%)$ and $26(26 \%)$ of cases and controls of whom $94 \%$ and $16 \%$ were found to practice physical activities for less than one hour per week. This means that $85 \%$ of controls practice physical activities for more than one hour per week, and this have showed statistical significant difference $(\mathrm{P}<0.0001)$.

On the other hand, $43.3 \%$ and $57.7 \%$ of the cases have attended the menarge at the age of less than 13 years and more than 14 years, respectively, as indicated in Fig. (2). Moreover, only $14.5 \%$ of the controls have attended the menarge at the age of 13 years old or less.

What's more, 59(39.3\%) of the cases and $12(12 \%)$ of controls have experienced menopause; of whom $27(18 \%)$ and $12(12 \%)$ have experienced menopause at the age range 41-50 years, respectively, while $12(8.0 \%)$ of the cases have experienced menopause at the age range 51 or more. 


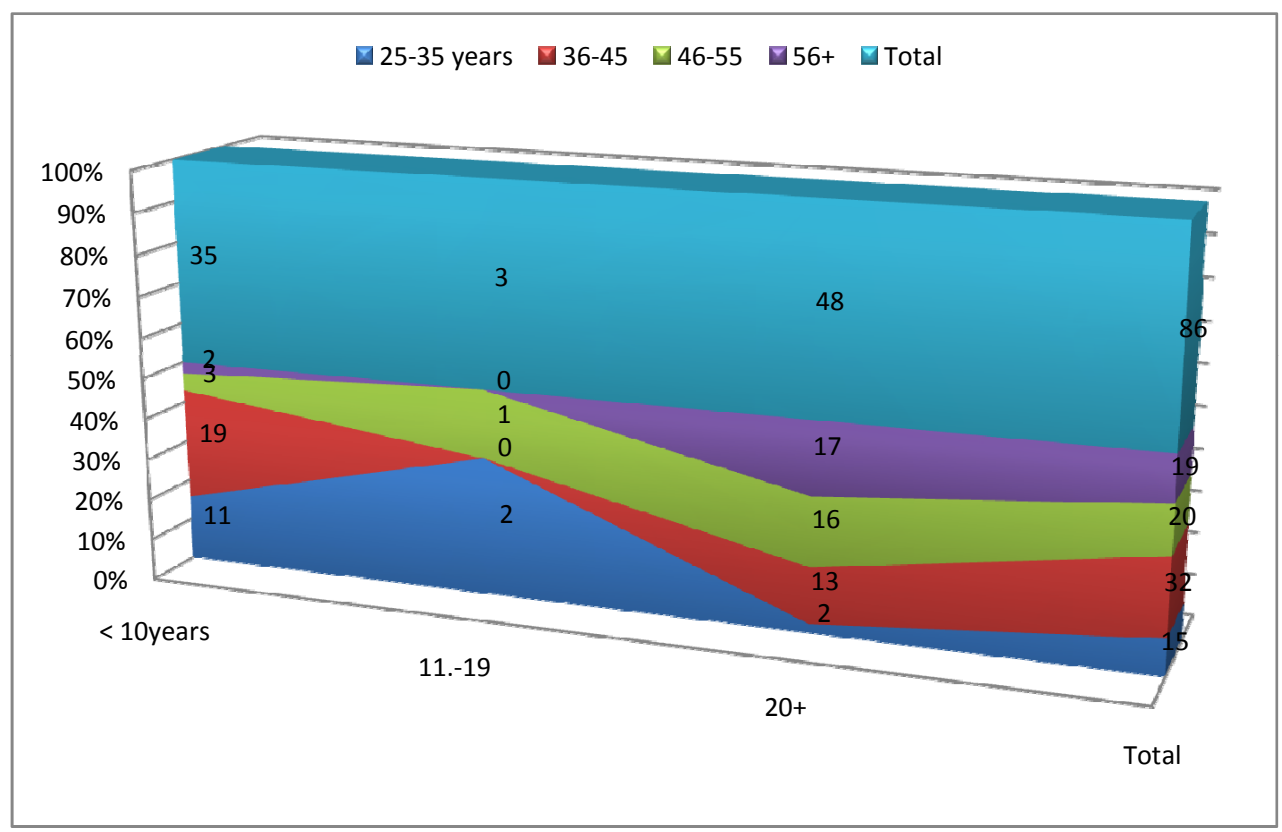

Fig. (1). Period being over weight in years by age.

Concerning marital status, $16(10.7 \%)$ of the cases and $8(8 \%)$ of the controls were unmarried $(\mathrm{P}<0.002)$, while $103(68.7 \%)$ and $92(92 \%)$ were married, widows or divorced, respectively.

Out of 150 cases and 100 controls 112(74.7\%) and $83(83 \%)$ delivered first alive birth, of whom $63(56.3 \%)$ and $31(31 \%)$ have delivered first alive birth at an age of 20 years or less, respectively. While the remaining $49(43.7 \%)$ and $52(52 \%)$ of the cases and controls have delivered first alive birth at an age range of 21 years and more.
On the other hand, $23.3 \%$ of the cases and $4 \%$ of the controls had no children, while $71.8 \%$ and $61 \%$ of had 5 and less number of children, respectively. However, 43(28.6\%) of the cases and $20(20 \%)$ of the controls had more than 5 numbers of children. The risk of breast cancer increase with the decreased number of children $(\mathrm{P}<0.002)$.

The majority of the patients $114(76.0 \%)$ were house women, while teachers, employee, Farmers, Police women, Sellers, students and mid wives constituted $4.7 \%, 7.3 \%$, $6.7 \%, 2.7 \%, 1.3 \%, 0.7 \%$ and $0.7 \%$ respectively, as shown in

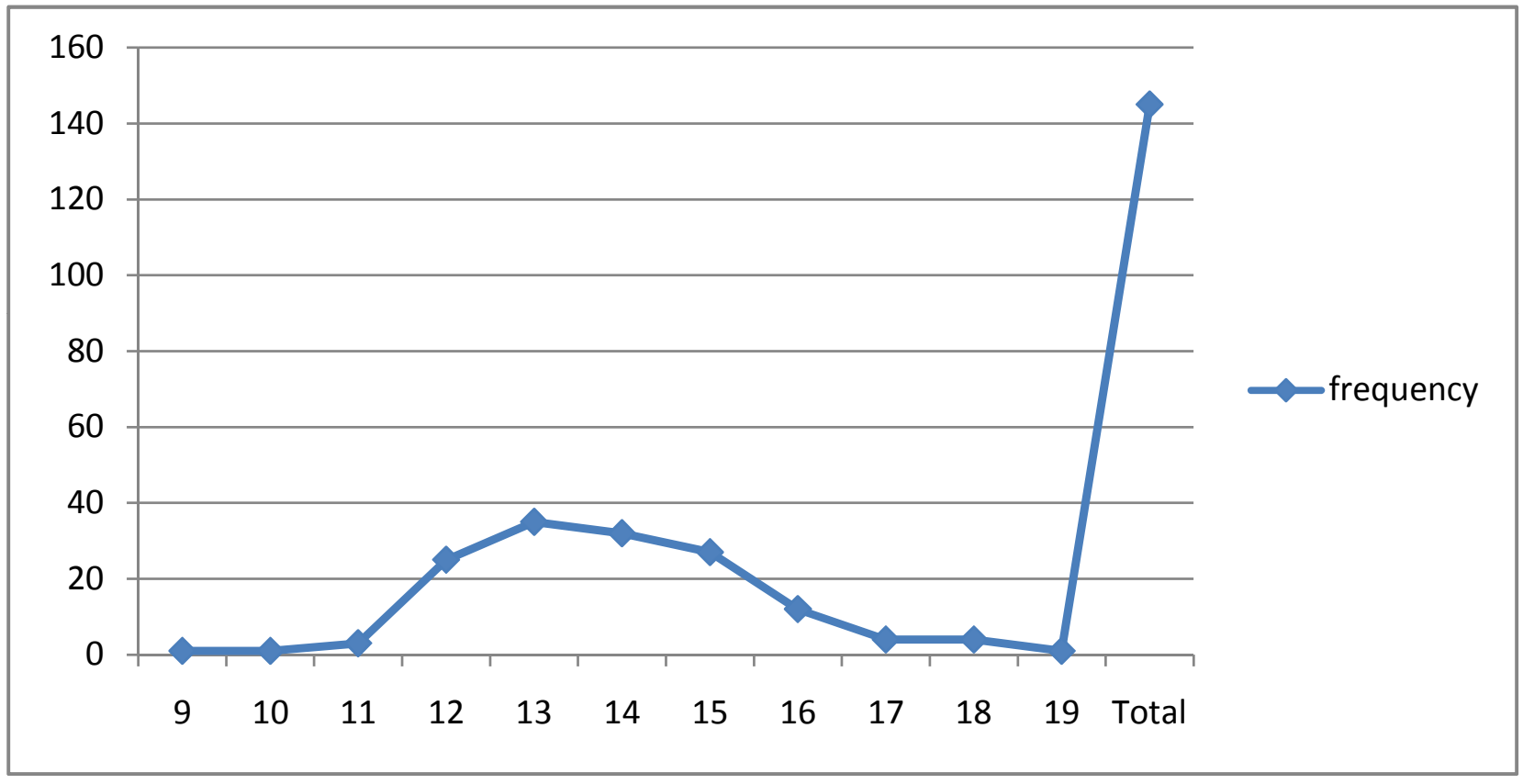

Fig. (2). Description of the study population by age at menarge. 

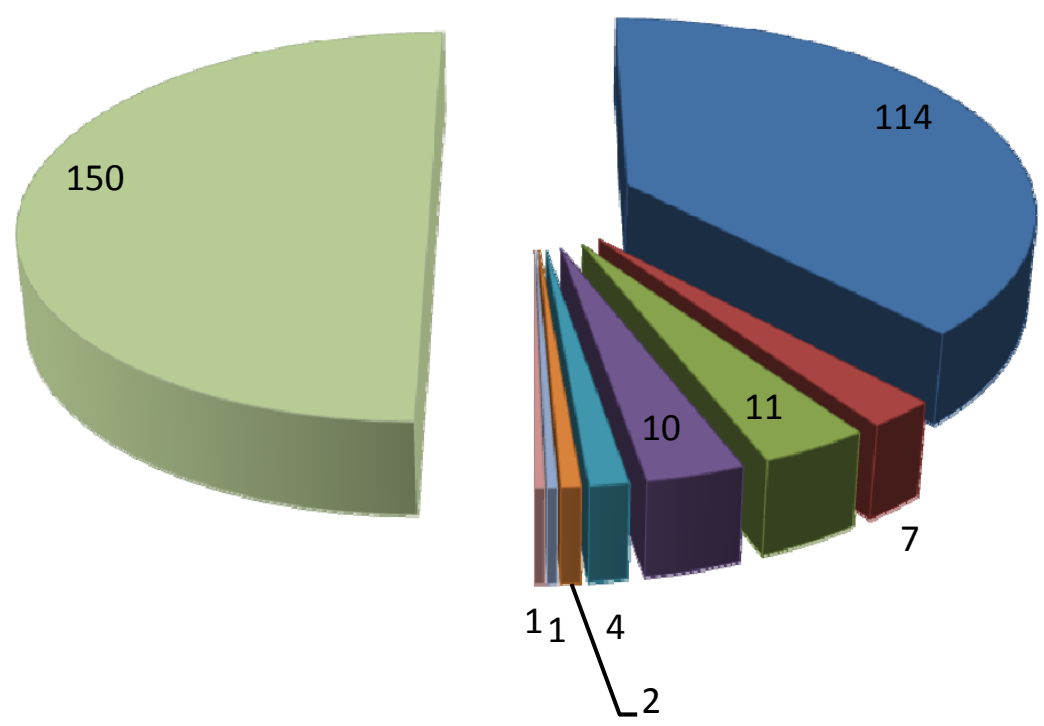

House keeper

Teacher

Employee

Farmer

Police woman

Seller

Student

Mid wife

Total

Fig. (3). Description of the study population by occupation.

Fig. (3). For the controls, house women represent $47 \%$, while teachers, employee, Farmers, Police women, Sellers, students and mid wives constituted 4\%,39\%, 1\%, 0\%, 1\%, $1 \%$ and $0 \%$, respectively.

Breast cancer was distributed more frequently among Gaalyaeen tribes 61(40.7\%), while, tribes of Gohina, Koahla, Bija, African group and Nubian, constituted 20.7\%, 5.3\%, $1.3 \%, 14.7 \%$ and $17.3 \%$ respectively, as shown in Fig. (4).

Furthermore, $37.3 \%$ of the patients were from Khartoum state, $20.0 \%$ from Northern States, $19.3 \%$ from Western Sudan, $15.3 \%$ from Central province, and the rest $(8.0 \%)$ from Eastern Sudan, as shown in Fig. (5).

\section{DISCUSSION}

Female breast cancer is by far the leading cancer in the Sudan, which has been recognized as an important health problem that associated with a high rate of mortality and morbidity. Percentages of the breast cancers represented $38.4 \%$ to $42 \%$ of all female cancers [1].

Knowledge of risk factors, as well as, rising of the awareness are momentous, particularly in a country like the Sudan, where many patients present from remote areas with poor health services. For that reason, the incidence and mortality of breast cancer are high, remarkably constant and the frequency is increasing particularly amongst younger women. However, the current study was an attempt to find

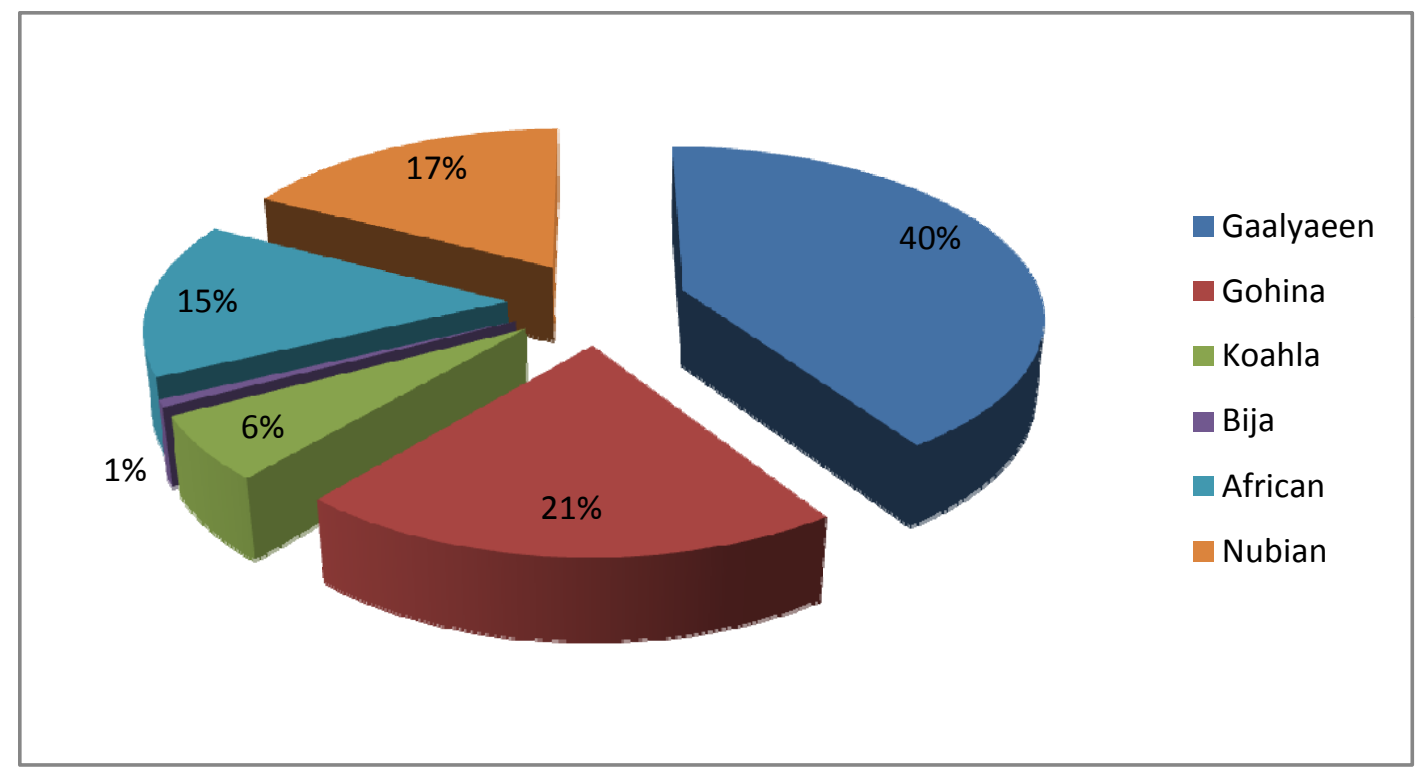

Fig. (4). Description of the cases by ethnic groups. 

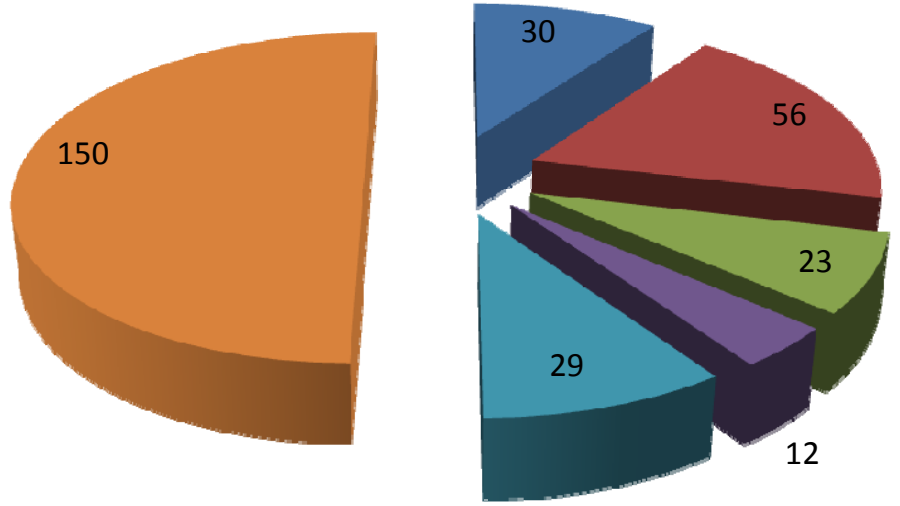

North

Khartoum

Centre

East

West

Total

Fig. (5). Description of the study population by residence (state).

out the frequencies of exposure to some risk factors that might be associated with the breast cancer in the Sudan.

Exposure to endogenous estrogens increases the risk of breast cancer. Women who start menstruating before age 12 or begin menopause after age 55 generally have more monthly cycles and therefore a longer lifetime exposure to estrogen. This tends to increase their risk of breast cancer. Women who have never had children or who start their families late also have a higher lifetime exposure to estrogens. In the current study, and out of the 150 patients with breast cancer, $38(25.3 \%)$ were Identified as having a previous history of oral contraceptives usage, as well as, $38(38 \%)$ of the controls were users. Although, this study was not a cohort study (e.g. no follow up), these variables clearly signify the risk of breast cancer in the Sudan.

Multiple epidemiological studies have been carried out to investigate the association between serum sex hormones and premenopausal breast cancer risk, but despite the strength of the sex hormone paradigm in breast cancer etiology, the results have been equivocal $[4,5]$.

The limitations of our study include its not being a cohort design and the fact that the results are based on frequency measurements in a history reminder. Such measurements provide an imperfect estimate of an individual woman's long-term oral contraceptives usage.

Furthermore, Breast lesions increased the levels of serum progesterone and this was found to be statistically significant $\mathrm{P}<0.03$. Progesterone's role in breast cancer is controversial. It has been hypothesized that its activity of opposing estrogenic stimulation of the breast decreases breast cancer risk [6]. On the other hand, some believe that the risk of breast cancer is increased because breast mitotic rates are highest in the luteal (high progesterone) phase of the menstrual cycle [7]. Maurine study have demonstrated that apoptosis in the mammary gland is inhibited by progesterone [8] and that the progesterone signal contributes to mammary tumors susceptibility [5].
Experimental studies have shown that estrogens have a proliferative effect on breast tissue [9] are probably related to increase mitotic activity, and are believed to be a promoting influence rather than an initiating effect [10]. The proliferation of cells is essential for carcinogenesis because the risk of errors during DNA replication is increased during cell division, which if not corrected, can lead to cancer [11]. Although, the results of our study were consistent among cases and controls, other studies have shown that estrogen is believed to increase the risk of breast cancer [7, 12].

In regard to the family history of breast cancer, of the 150 breast cancer patients, $22(14.7 \%)$ were found with a family history of breast cancer [13]. conducted a systematic review by searching MEDLINE and CENTRAL databases for evidence (in all languages) published in peer-reviewed journals from 1966 to July 2008 that provided estimates of breast cancer risk according to family history. Twelve articles were identified and the quality of each study was assessed using the United States Preventive Services Task Force grading system. Results from 10 studies and one pooled analysis of 54 studies suggest that the use of OCs does not significantly modify the risk of breast cancer among women with a familial history of breast cancer; however, evidence from some studies shows that some women may be at a greater risk. Women with a family history of breast cancer are at increased risk of the disease, but no study has been large enough to characterize reliably how, over women's lives, this risk is influenced by particular familial patterns of breast cancer. In a report, on the relevance of breast cancer in first-degree relatives is based on combined data from 52 epidemiological studies. Individual data on breast cancer in first-degree relatives (mothers, sisters, and daughters) of 58209 women with breast cancer and of 101986 controls were collected, checked, and analyzed centrally. Altogether $7496(12.9 \%)$ women with breast cancer and $7438(7.3 \%)$ controls reported that one or more first-degree relatives had a history of breast cancer: $12 \%$ of women with breast cancer had one affected relative and 1\% had two or more. Risk ratios for breast cancer increased with increasing numbers of affected first-degree relatives. Eight 
out of nine women who develop breast cancer do not have an affected mother, sister, or daughter. Although, women who have first-degree relatives with a history of breast cancer are at increased risk of the disease, most will never develop breast cancer, and most who do will be aged over 50 when their cancer is diagnosed. In countries where breast cancer is common, the lifetime excess incidence of breast cancer is $5.5 \%$ for women with one affected first-degree relative and $13.3 \%$ for women with two [14].

In the current study, $26(17.3 \%),(\mathrm{P}<0.04)$ of patients have revealed a past history of benign breast disease. Benign breast lesions are quite heterogeneous but of these, the best characterized premalignant lesions are atypical ductal hyperplasia, atypical lobular hyperplasia and lobular carcinoma in situ. Prior studies of benign breast disease have focused on the risks for subsequent breast cancer associated with benign lesions classified into one of three broad histopathologic categories: nonproliferative, proliferative, or proliferative with atypia. What remains uncertain, however, is whether the absolute number of benign lesions predicts a malignant outcome. To address this, Worsham et al. [15] examined data from a cohort of 4,544 patients from which $4.5 \%(\mathrm{n}=201)$ developed breast cancer during an average follow-up period of 10.3 years.

Conversely, $21(14 \%),(\mathrm{P}<0.07)$, of the studied subjects in the current study have revealed that they have done previous breast biopsies. In a cohort study by Cheng et al. [16], $9.21 \%$ of preoperative nonmalignant papillary lesions were converted to malignant diagnosis after surgery. Atypical lesions and patients aged 45 years or older were significant factors associated with such conversion. Out of the 150 women with breast cancer, 20 (13.3\%) were detected as having a previous history of breast cancer and the remaining $130(86.7 \%)$ without previous history of breast cancer. Several studies have evaluated the effect of a supplementary dose of radiation to the tumor bed on the rates of local recurrence among patients who received radiotherapy after breast-conserving surgery for early breast cancer. Age no more than 35 years and the presence of an extensive intraductal component are associated with an increased risk of local recurrence after breast-conserving therapy. Vascular invasion causes a higher risk of local recurrence after mastectomy as well as after breastconserving therapy and should therefore not be used for deciding between the two treatments $[17,18]$. The response rate to therapy in patients with recurrence more than 10 years after surgery is high, and such patients have significantly better prognosis compared with other patients with recurrence, which means that aggressive therapy may be appropriate management for patients with very late recurrence [19].

Moreover, in this study, 11(7.3\%) patients have claimed other cancers in their families and $86(57.3 \%)$ have confessed their being over weighed in most of their lives. Lacey et al. [20] evaluated known risk factors for breast cancer in a cancer screening trial that does not have breast cancer as a study endpoint but is large enough to provide numerous analytic opportunities for breast cancer. They evaluated risk factors for breast cancer $(\mathrm{N}=2085)$ among 70,575 women who were randomized in the Prostate, Lung, Colorectal and Ovarian Cancer Screening Trial. As expected, increasing age, nulliparity, positive family history of breast cancer, and use of menopausal hormone therapy were positively associated with breast cancer. Later age at menarche ( 16 years or older $v s<12$ : RR $=0.81,95 \% \mathrm{CI}$, $0.65-1.02$ ) or menopause (55 years or older $v s<45$ : $\mathrm{RR}=$ $1.29,95 \%$ CI, 1.03-1.62) were less strongly associated with breast cancer than was expected. There were weak positive associations between taller height and heavier weight, and only severe obesity [body mass index (BMI; $\mathrm{kg} / \mathrm{m}(2)) 35$ or more vs 18.5-24.9: $\mathrm{RR}=1.21,95 \% \mathrm{CI}, 1.02-1.43]$ was statistically significantly associated with breast cancer. These findings offers continued opportunities for new breast cancer investigations, but these analyses suggest that the associations between breast cancer and age at menarche, age at menopause, and obesity might be changing as the underlying demographics of these factors change. However, in the current study, $44.8 \%$ and $66.9 \%$ have attended the menarge at the age of 13 and 14 respectively. Moreover, only $14.5 \%$ have attended the menarge at the age of 15 years old or more.

In regard to the exposure to pesticides, $72(48 \%)(\mathrm{P}<$ 0.01 ) of the study subjects have confessed a pesticides exposure; also exposure to plasticizers was approved among $65(43.3 \%)(\mathrm{P}<0.04)$, of the studied subjects. Several reports have since 15 years reported positive correlations between blood or peritumoral adipose tissue levels of persistent organic compounds including organochloride pesticides and breast cancer risk. Moreover, fetal or perinatal exposition to low doses of such endocrine disruptors induces premalignant or malignant transformation of adult mammary gland in rodents [21]. The association between pesticide use and breast cancer incidence was examined among farmers' wives in a large prospective cohort study Breast cancer standardized incidence ratios were 0.87 (95\% confidence interval: $0.74,1.02)$ for women who reported ever applying pesticides and 1.05 (95\% confidence interval: $0.89,1.24)$ for women who reported never applying pesticides. There was some evidence of increased risk associated with use of 2,4,5trichloro-phenoxypropionic acid $(2,4,5-\mathrm{TP})$ and possibly use of dieldrin, captan, and 2,4,5-trichlorophenoxyacetic acid (2,4,5-TP), but small numbers of cases among those who had personally used the pesticides precluded firm. There was no clear association of breast cancer risk with farm size or washing of clothes worn during pesticide application, but risk was modestly elevated among women whose homes were closest to areas of pesticide application [22]. Since the percentage of those exposed is very high in the present study, further follow-up of such cohort should help clarify the relation between pesticide exposure and breast cancer risk.

Butyl benzyl phthalate (BBP) is a plasticizer commonly used in pipes, vinyl floor tiles, vinyl foams, and carpet backing, and to a minor extent, in cellulose plastics and polyurethane 9. This compound has shown to be weakly estrogenic [23] and to induce adverse effects on the development of male reproductive system; thus acting as an endocrine disruptor. BBP has shown endocrine-disrupting properties, thus having a potential effect on hormonesensitive tissues [24].

Out of the 150 women, 44 (29.3\%) were found to have a previous physical activity and the remaining $106(70.7 \%)$ did 
not practice and mean of physical activities $(\mathrm{P}<0.0001)$. BRCA1/2 mutation carriers have a high lifetime risk of developing breast cancer. Differences in penetrance indicate that this risk may be influenced by lifestyle factors. Because physical activity is one of the few modifiable risk factors, it may provide a target to add to breast cancer prevention in this high-risk population. The association between selfreported lifetime sports activity and breast cancer risk was examined in a nationwide retrospective cohort study, including 725 carriers, of whom 218 had been diagnosed with breast cancer within 10 years prior to questionnaire completion. A non-significantly decreased risk for ever engaging in sports activity was found to be $(\mathrm{HR}=0.84$, $95 \% \mathrm{CI}=0.57-1.24)$. Among women who had participated in sports, a medium versus low level of intensity and duration (i.e., between 11.0 and 22.7 mean MET hours/week averaged over a lifetime) reduced the risk of breast cancer $(\mathrm{HR}=0.59$, $95 \% \mathrm{CI}=0.36-0.95$ ); no dose-response trend was observed. For mean hours/week of sports activity, a non-significant trend was observed $(\mathrm{HR}$ (low versus never $)=0.93,95 \% \mathrm{CI}=$ $0.60-1.43$; $\mathrm{HR}$ (medium versus never) $=0.81,95 \% \mathrm{CI}=0.51$ 1.29; $\mathrm{HR}$ (high versus never) $=0.78,95 \% \mathrm{CI}=0.48-1.29 ; \mathrm{p}$ $($ trend overall $)=0.272 ; \mathrm{p}($ trend active women $)=0.487)$. For number of years of sports activity no significant associations were found. Among women active in sports before age 30, mean MET hours/week showed the strongest inverse association of all activity measures (HR (medium versus low) $=0.60,95 \% \mathrm{CI}=0.38-0.96$; HR(high versus low $)=$ $0.58,95 \% \mathrm{CI}=0.35-0.94 ; \mathrm{p}$ (trend) $=0.053$ ). Engaging in sports activity after age 30 was also inversely associated with breast cancer risk $(\mathrm{HR}=0.63,95 \% \mathrm{CI}=0.44-0.91)$. These findings results indicate that sports activity may reduce the risk of breast cancer in BRCA1/2 mutation carriers [25]. Concerning the distribution of the study population by residence, $37.3 \%$ of the patients were from Khartoum state, $20.0 \%$ were from Northern states, $19.3 \%$ from Western Sudan. However, similar distribution was previously reported by Hussain et al. (2010). They reported that $77 \%$ of the breast cancer cases were from Khartoum state, $13 \%$ were from East, $7 \%$ were from western Sudan and $3 \%$ were from Northern states.

In regard to the distribution of study population by race (tribe). Breast cancer was found to occur more frequently among Gaalyaeen tribes 61(40.7\%), while, tribes of Gohina, Koahla, Bija, African group and Nubian, constituted 20.7\%, $5.3 \%, 1.3 \%, 14.7 \%$ and $17.3 \%$ respectively. These findings supported the study by Hussain et al. [26]. They found that the majority of cases were among Gaalyaeen tribe followed by Nubian.

In conclusion, there was variable exposure to many risk factors for breast cancer in the Sudan. The study suggests further separate measurement of factors such as, the age at the first child birth, diet, marital status, family history of the breast cancer, the use of hormonal contraceptive pills, and physical activities etc. Cohort mean of evaluation is highly recommended.

\section{REFERENCES}

[1] Ahmed HG, Hussein MO. Preoperative predictive values of serum CA15-3 and CEA within Sudanese women with breast cancer. Sudan JMS 2009; 4(2): 63-5.

[2] Cavalieri E, Chakravarti D, Guttenplan J, et al. Catechol estrogen quinones as initiators of breast and other human cancers: implications for biomarkers of susceptibility and cancer prevention. Biochim Biophys Acta 2006; 1766(1): 63-78.

[3] Madigan MP, Ziegler RG, Benichou J, Byrne C, Hoover RN. Proportion of breast cancer cases in the United States explained by well-established risk factors. J Natl Cancer Inst 1995; 87: 1681-5.

[4] Key TJ, Pike MC. The role of oestrogens and progestogens in the epidemiology and prevention of breast cancer. Eur J Cancer Clin Oncol 1988; 24: 29-43.

[5] Haslam SZ, Osuch JR, Raafat AM, Hofseth LJ. Postmenopausal Hormone replacement therapy: effects on normal mammary gland in humans and in a mouse postmenopausal model. J Mammary Gland Biol Neoplasia 2002; 7: 93-105.

[6] Foidart JM, Colin C, Denoo X, et al. Estradiol and progesteroneregulate the proliferation of human breast epithelial cells. Fertil Steril 1998; 69: 963-9.

[7] Ho CCK, Rohaizak M, Zulkifli SZ, Siti-Aishah MA, Nor-Aini U, Sharifah-Noor-Akmal SH. Serum sex hormone levels in pre- and postmenopausal breast cancer patients. Singapore Med J 2009; 50(5): 513-8.

[8] Haslam SZ, Counterman LJ. Mammary stroma modulates hormonal responsiveness of mammary epithelium in vivo in the mouse. Endocrinology 1991; 129: 2017-23.

[9] David BT. Do hormones cause breast cancer? Cancer 1984; 53(3): 595-604.

[10] Telang NT, Katdare M, Bradlow HL, Osborne MP. Estradiol metabolism: an endocrine biomarker for modulation of human mammary carcinogenesis. Environ Health Prospect 1997; 105(3): 559-64.

[11] Dixon K, Kopras E. Genetic alterations and DNA repair in human carcinogenesis. Semin Cancer Biol 2004; 14(6): 441-8.

[12] Kaaks R, Berrino F, Key $\mathrm{T}$, et al. Serum sex steroids in premenopausal women and breast cancer risk within the European Prospective Investigation into Cancer and Nutrition (EPIC). J Natl Cancer Inst 2005; 97(10): 755-65.

[13] Gaffield ME, Culwell KR, Ravi A. Oral contraceptives and family history of breast cancer. Contraception 2009; 80(4): 372-80.

[14] Collaborative Group on Hormonal Factors in Breast Cancer. Familial breast cancer: collaborative reanalysis of individual data from 52 epidemiological studies including 58,209 women with breast cancer and 101,986 women without the disease. Lancet 2001; 358(9291): 1389-99.

[15] Worsham MJ, Raju U, Lu M, Kapke A, Cheng J, Wolman SR. Multiplicity of benign breast lesions is a risk factor for progression to breast cancer. Clin Cancer Res 2007; 13: 5474-9.

[16] Cheng TY, Chen CM, Lee MY, et al. Risk Factors Associated with Conversion from Nonmalignant to Malignant Diagnosis After Surgical Excision of Breast Papillary Lesions. Ann Surg Oncol 2009; 16(12): 3375-9.

[17] Vrieling C, Collette L, Fourquet A, et al. The influence of patient, tumor and treatment factors on the cosmetic results after breastconserving therapy in the EORTC "boost vs no boost" trial. Radiother Oncol 2000; 55: 219-32.

[18] Voogd AC, Nielsen M, Peterse JL, et al. Differences in risk factors for local and distant recurrence after breast-conserving therapy or mastectomy for stage I and II breast cancer: pooled results of two large European randomized trials. J Clin Oncol 2001; 19: 1688-97.

[19] Takeuchi H, Muto Y, Tashiro H. Clinicopathological characteristics of recurrence more than 10 years after surgery in patients with breast carcinoma. Anticancer Res 2009; 29(8): 3445-8.

[20] Lacey JV Jr, Kreimer AR, Buys SS, et al. Prostate, Lung, Colorectal and Ovarian (PLCO) Cancer Screening Trial Project Team. Breast cancer epidemiology according to recognized breast cancer risk factors in the Prostate, Lung, Colorectal and Ovarian (PLCO) Cancer Screening Trial Cohort. BMC Cancer 2009; 9: 84. 
[21] Fénichel P, Brucker-Davis F. Environmental endocrine disruptors and breast cancer: new risk factors? Gynecol Obstet Fertil 2008; 36(10): 969-77.

[22] Lawrence SE, Deirdre AH, Jane AH, et al. Pesticide Use and Breast Cancer Risk among Farmers' Wives in the Agricultural Health Study. Am J Epidemiol 2005; 161(2): 121-35.

[23] Okubo T, Suzuki T, Yokoyama Y, Kano K, Kano I. Estimation of estrogenic and anti-estrogenic activities of some phthalate diesters and monoesters by MCF-7 cell proliferation assay in vitro. Biol Pharm Bull 2003; 26(8): 1219-24.
[24] Moral R, Wang R, Russo IH, Mailo DA, Lamartiniere CA, Russo J. The plasticizer butyl benzyl phthalate induces genomic changes in rat mammary gland after neonatal/prepubertal exposure. BMC Genomics 2007; 8: 453.

[25] Pijpe A, Manders P, Brohet RM, et al. Physical activity and the risk of breast cancer in BRCA1/2 mutation carriers. Breast Cancer Res Treat. Breast Cancer Res Treat 2010; 120(1): 235-44.

[26] Ahmed HG, Ali AS, Almobarak AO. Frequency of breast cancer among Sudanese Patients with breast palpable lumps. Indian J Cancer 2010; 47(1): 48-51.

(C) Ahmed et al.; Licensee Bentham Open.

This is an open access article licensed under the terms of the Creative Commons Attribution Non-Commercial License (http://creativecommons.org/licenses/by-nc/ 3.0/) which permits unrestricted, non-commercial use, distribution and reproduction in any medium, provided the work is properly cited. 